\title{
Minute Hyalinizing Trabecular Tumor Misinterpreted as a Blood Vessel on Ultrasonography: A Case Report
}

\section{(a) (2) (1) $\otimes_{\text {Version } 1}$}

\section{Introduction}

Hyalinizing trabecular tumor (HTT) of the thyroid is a rare tumor of follicular cell origin with a trabecular growth pattern and marked intratrabecular hyalinization [Carney JA, et al. Am J Surg Pathol 2008; 32: 1877-1889]. On fine needle aspiration cytology (FNAC), it is often interpreted as papillary thyroid carcino$\mathrm{ma}$ (PTC) because of the presence of intranuclear cytoplasmic inclusions [Kuma S, et al. Acta Cytol 2003; 47: 399-404]. Few reports have described ultrasound findings of HTT [Kobayashi K, et al. ] Med Ultrasonics 2007; 34: 43-47; Choi WJ, et al. Acta Radiol 2015; 56: 1113-1118; LeeS, et al. Thyroid 2011;21: 253-259]. Reportedly, the findings were similar to follicular adenoma. Recently, we encountered a case of minute HTT that was incidentally observed by ultrasonography and interpreted as a blood vessel. To the best of our knowledge, our case is the smallest HTT detected by ultrasound examination. We describe herein the ultrasound findings of a minute HTT that we experienced and discuss the significance of detecting such a lesion.

\section{Case}

A 44-year-old woman visited our hospital because of an evaluation of thyroid nodules detected by medical check. On physical examination, the nodules were not palpable. Laboratory results, including those from a thyroid hormone test, were within normal limits. Ultrasound examination revealed one nodule in her right lobe and 3 nodules in the left lobe of the thyroid. Since the nodule of the right lobe was interpreted as a blood vessel, FNAC was not performed. The 2 nodules located in the left lobe measured 11 and $9 \mathrm{~mm}$ in diameter, and both were diagnosed as PTC by FNAC. The remaining one was a cyst. Lymph node enlargement suggesting metastasis was noted in the left neck. Subsequently, total thyroidectomy with a left modified radical neck dissection was performed. Microscopic examination confirmed the diagnoses of PTC of the left 2 nodules and metastatic PTC of the lymph nodes. The right nodule was a hyalinizing trabecular tumor.

\section{Ultrasound Findings}

Ultrasound examination was performed using a Toshiba APLIO 500 TUS-A500 ultrasound system with PLT-1005BT (10 MHz) linear probes. B-mode image revealed the hypoechoic nodule located in the right lobe measuring $2 \mathrm{~mm}$ in diameter ( $\triangleright \mathbf{F i g . ~ 1 a , ~ b ) . ~}$ It was oval in shape and isolated. The margin was well-defined but slightly rough. It was not associated with a hypoechoic halo. The echotexture was homogeneous. There was no calcification. Power Doppler image showed intensive intratumoral blood flow ( Fig. 1c). In the left lobe, 3 nodules were detected. 2 of these nodules measured 11 and $9 \mathrm{~mm}$ in diameter, respectively. They were hypoechoic, and their margins were ill-defined and irregular ( $\triangleright$ Fig. 2). The remaining nodule was a cyst.

\section{Pathological Findings}

Grossly, nodule measuring 2 mm was present ( $\triangleright$ Fig. 3 ). The size and location of the nodule corresponded to the lesion detected by ultrasound examination, and no additional nodule was identified in the right lobe. Microscopically, it was circumscribed but not encapsulated. Tumor cells were polygonal or fusiform and showed a trabecular growth pattern. Intranuclear cytoplasmic inclusions, yellow bodies, and nuclear grooves were observed. The trabeculae were surrounded with periodic acid-Schiff (PAS)-positive basement membrane materials. The materials were also present at the intratrabecular area, and highlighted by PAS stain ( Fig. 4a). Immunohistochemically, the cell membrane was positive for Ki-67 (MIB1, 1:200 dilution, DAKO) ( $>$ Fig. 4b). The left 2 nodules that were diagnosed as PTC by FNAC were classic PTC. The cyst of the left lobe was an adenomatous nodule. There were no findings of chronic thyroiditis.

\section{Discussion}

HTT is a rare tumor of the thyroid. The tumor is characterized by a trabecular growth pattern, abundant intranuclear cytoplasmic inclusions, intratrabecular basement membrane materials, and cell membrane immunoreactivity for Ki-67
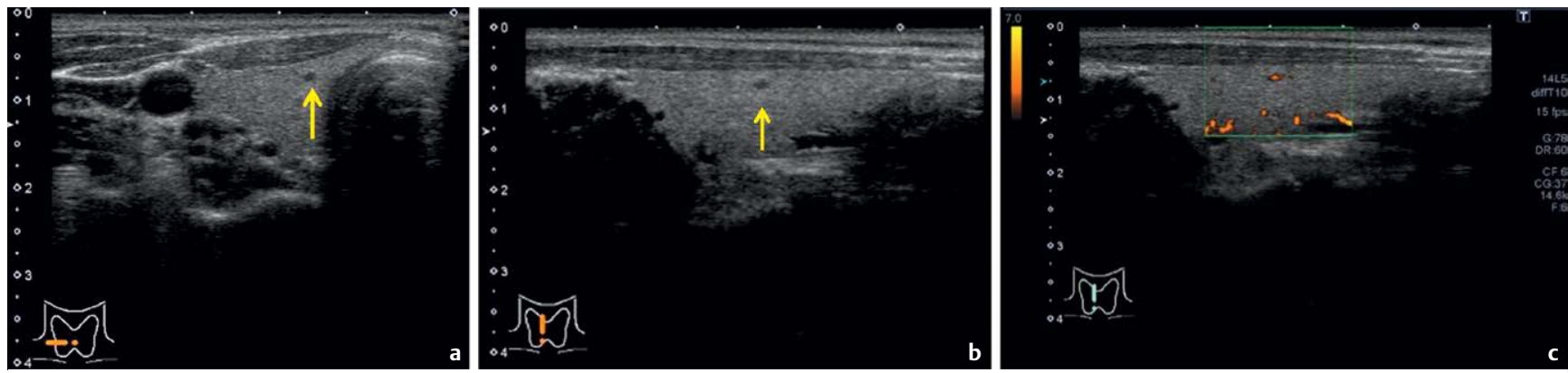

Fig. 1 On B-mode image, a small hypoechoic lesion (arrows) is seen in the right lobe of the thyroid (a a transverse view, $\mathbf{b}$ a longitudinal view).

Power Doppler image shows intensive intratumoral blood flow $\mathbf{c}$. 
[Hirokawa M, Carney JA. Am ] Surg Pathol 2000; 24: 575-578]. As the small lesion we reported was incidentally observed in the right lobe by ultrasound examination and expressed all of these characteristics, the diagnosis of HTT was made.

Few reports have investigated the ultrasound findings of HTT. In 2007, Kobayashi et al. reported a case of HTT, which was ultrasonographically characterized by an irregular shape, a delicately jagged border, hypoechoic and heterogeneous internal echoes, and very rich intratumoral blood flow [Kobayashi K, et al. ] Med Ultrasonics
2007; 34: 43-47]. They proposed the term "tumor inferno" for the last finding. In 2011, Lee et al. reported the ultrasound findings of 10 HTT cases [Lee S, et al. Thyroid 2011;21:253-259]. The tumors were oval-to-round, well-defined, solid, and hypoechoic. $70 \%$ of the cases showed a heterogeneous echotexture. None revealed calcifications. The ultrasound findings of 24 HTT cases reported by Choi et al. in 2014 [Choi W], et al. Acta Radiol 2015; 56: 11131118] were mostly similar to those reported by Lee et al. However, they reported 7 cases (29.2\%) having malignant features

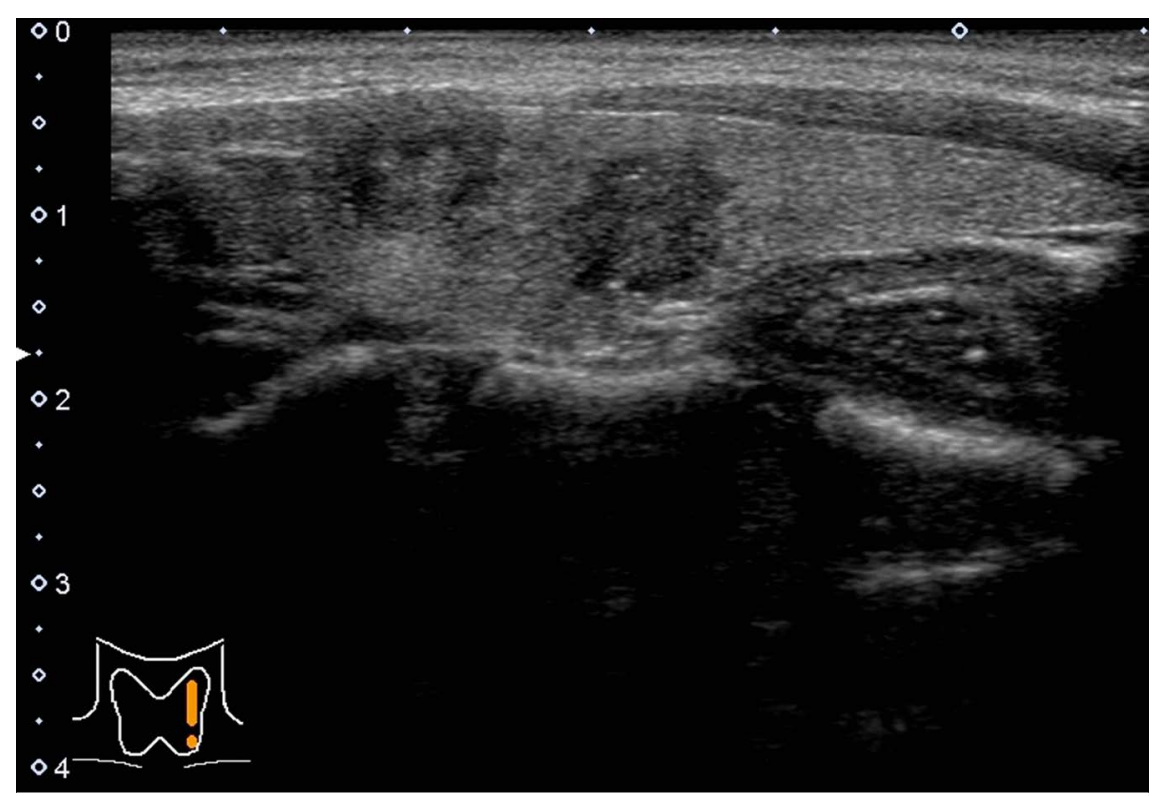

- Fig. 2 On B-mode image, 2 nodules are seen in the left lobe of the thyroid. They are hypoechoic, and their margins are ill-defined and irregular. such as marked hypoechogenicity and spiculated margins. In addition, they described the findings of peri- and/or intranodular vascularity. The ultrasound findings characteristic of HTT seem to be solid, hypoechoic, and vascular, and our case revealed them even though the tumor was minute. According to the 2015 American Thyroid Association Management Guidelines for Adult Patients with Thyroid Nodules and Differ-

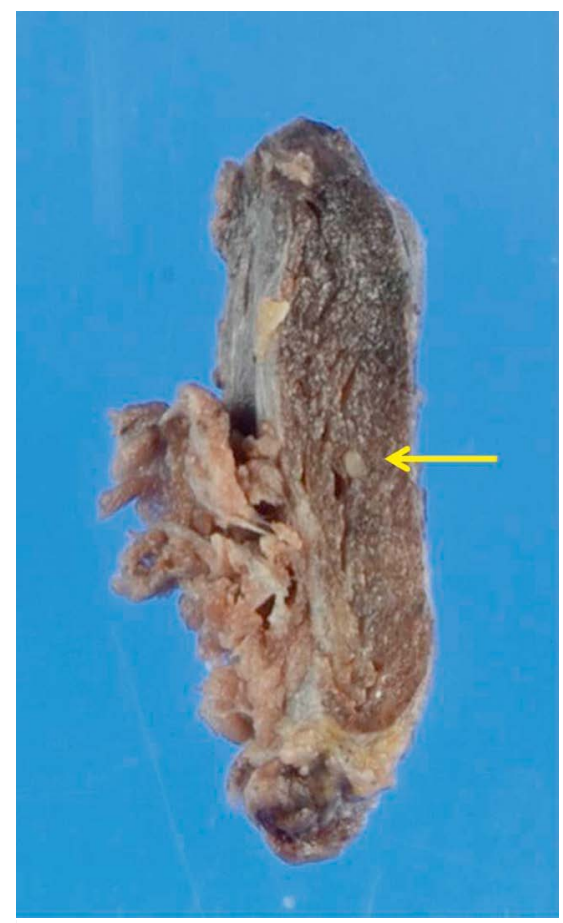

- Fig. 3 Cut surface of the resected right lobe. A solid and well-demarcated nodule measuring $2 \mathrm{~mm}$ is seen (arrow).
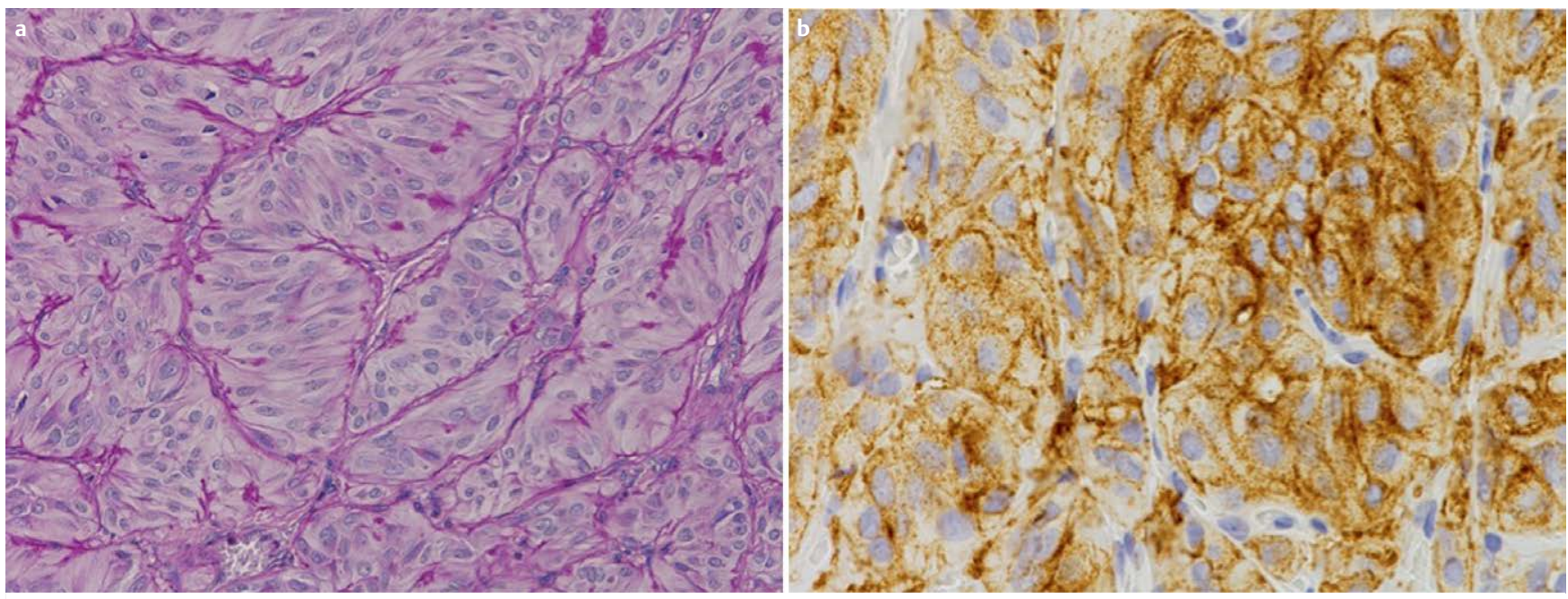

- Fig. 4 Tumor cells showing a trabecular growth pattern. Peritrabecular and intratrabecular hyaline materials are highlighted by periodic acid

- Schiff stain $\mathbf{a}$. The cell membrane is immunohistochemically positive for Ki-67 b. 
entiated Thyroid Cancer [Haugen BR, et al. Thyroid 2016; 26: 1-133], the nodules with such ultrasound features are classified as "intermediate suspicion", and the risk of malignancy is estimated to be $10-20 \%$, but FNAC is recommended for $>1.0 \mathrm{~cm}$.

The HTT we presented was $2 \mathrm{~mm}$ in diameter and was much smaller than those previously reported in the English literature. To the best of our knowledge, all the reported HTT cases with ultrasound findings were $>6 \mathrm{~mm}$. According to reports [Kobayashi K, et al. ] Med Ultrasonics 2007; 34: 43-47; Choi WJ, et al. Acta Radiol 2015; 56: 1113-1118; Lee S, et al. Thyroid 2011; 21: 253-259], ultrasound findings of HTT are similar to those of follicular adenoma, but they may not always be true depending on the nodular size. Particularly, small-sized ones like in our case are unexpected. In our case, it was interpreted as a blood vessel, because the blood flow was intensive and occupied throughout the small-sized tumor. An intrathyroidal blood vessel, especially large ones or vessels with abundant blood flow, may be observed as a low echoic lesion on B-mode ultrasonogram, and be confirmed by color Doppler image. The shape is typically linear but can be spotted like in our case. As strain elastography is a useful tool for thyroid lesion differentiation and seems to be serviceable even in small nodules [Cosgrove D, et al. Ultraschall Med 2013; 34: 238-253], the procedure, if carried out, might suggest the possibility of a tumor. It seems to be difficult to distinguish small HTT lesions from blood vessels, and other non-specific similar findings are very common. As a small-sized lesion is not an indication for FNAC (Haugen BR. et al. Thyroid 2016; 26: 1-133), it may not be necessary to observe in detail or to require follow-up. In conclusion, we assert that a minute HTT may mimic a blood vessel.

\section{Conflict of Interest}

We have no conflict of interest related to this article.

\section{Ethics Statement}

Our study was approved by the ethics committee of our hospital, and the subject gave informed consent.
Authors

Kumiko Tajiri' ${ }^{1}$, Mitsuyoshi Hirokawa², Maki Oshita', Hisashi Ota' ${ }^{1}$, Ayana Suzuki', Kaoru Kobayashi ${ }^{3}$, Hirotoshi Nakamura ${ }^{4}$, Akira Miyauchi ${ }^{3}$

1 Department of Laboratory Science, Kuma Hospital, Kobe, Japan

2 Department of Diagnostic Pathology and Cytology, Kuma Hospital, Kobe, Japan

3 Department of Surgery, Kuma Hospital, Kobe, Japan

4 Department of Internal Medicine, Kuma Hospital, Kobe, Japan

\section{Correspondence}

Kumiko Tajiri, MT

Department of Laboratory

Kuma Hospital

8-2-35, Shimoyamate-dori, Chuo-ku

650-0011 Kobe

Japan

Tel.: + 81/78/3713721

tajiri01@m.kuma-h.or.jp

Bibliografie

DOI http://dx.doi.org/10.1055/s-0042-121804

Published online: 2017 | Ultrasound Int Open

2017; 3: E43-E45

(c) Georg Thieme Verlag KG Stuttgart · New York ISSN 0722-1819 